\title{
Summary of Automation on the AURA 150-inch Telescope
}

\author{
D. L. CRAWFORD
}

Kitt Peak National Observatory* and Cerro Tololo Inter-American Observatory*

We hope to have a well-planned program tied to the astronomical specifications demanded. We also hope to save time and money, very important considerations in high cost, big science.

I have become really aware of the sink of dollars and man-hours that technology involves, especially so when it is not well defined and well planned. We hope to avoid a near bottomless sink if we can. Total systems design is very important, not just a careful design of each subsystem.

In adopting automation, I want to insist that we don't lock out certain experimental possibilities that we had before, or lock out certain type people (good ones) who are unwilling or unable to work with the highly sophisticated equipment we'll be providing. Excellent astronomy has been done with poor equipment used by good astronomers; even the best equipment doesn't do the best astronomy without the full involvement of good scientists. What I'm saying is, perhaps, that the system has to be our slave, not the reverse, and that we should not be limited by it.

What we want is an improvement in astronomical research, not merely in the gadgetry involved. The key to success may well be to strive for the best possible astronomer-technologist interface.

Now for a few details of our proposed system.

We have these constraints: (1) cost; (2) due to the highly transient nature of a large portion of our telescope users, the control features must not differ too markedly from current telescope-instrument systems, except perhaps to be simpler; (3) the change-over time from one telescope or instrument configuration to another should be minimal; (4) the mean-time-between-failures of the control system must be long and the mean-time-to-repair short, which implies simplicity in the design.

We propose: (1) a servo-controlled drive (see Fig. 1) with spur gearing, for increased efficiency and better linearity and dual opposed motors to have a balanced system. The increased efficiency and the use of unipolar (i.e. single-ended) amplifiers reduces the demands on amplifier design to the point that conservatively designed direct-coupled amplifiers may be used. Otherwise the design would have to depend on pulsed systems or on slow reacting systems. The first would tend to contribute to the electrical noise and the second would detract from the auto-guiding capability. (2) Separate absolute position encoding with resolution better than 1 arcsec and accuracy as good as the repeatability of the system will allow. Note that the absolute encoding link does not include any of the power transmission system up to the large gear, therefore, it does not include the hysteresis effects of the windup that occurs. (3) A dual computer system with a minimal set of high quality peripherals (see Fig. 2). This system will allow separation of data acquisition and "housekeeping" in separate cpu's to keep program interaction to a minimum. We will avoid using the processor consoles or the teletypewriter for routine control, but will put heavy reliance on labeled switches and push buttons. We will use removable patch boards to aid in changeover, and visual display via TV for information and data quality control.

We are now requesting prices for the system, and expect to sign contracts this autumn. We hope to have the system all in operation before the end of 1971 .

\section{DISCUSSION (Lasker and Crawford)}

J. Tinbergen: (a) How many telescopes do you have on the one computer at Cerro Tololo? $(b)$ How long did it take before you had a net gain, i.e. more observing time was gained by using the system than was lost by computer or peripheral failures? (c) How many hardware and software people do you have to keep on the spot to keep the whole thing running?

D. L. CRAWFORD: (a) There is one telescope on the system now but they intend to run two or three off that same system. We realize that there may be some interference between them. (b) The gain was significant even the first night, and has stayed significant all the way through. (c) The system at Cerro

* Operated by the Association of Universities for Research in Astronomy, Inc., under contract with the National Science Foundation. 


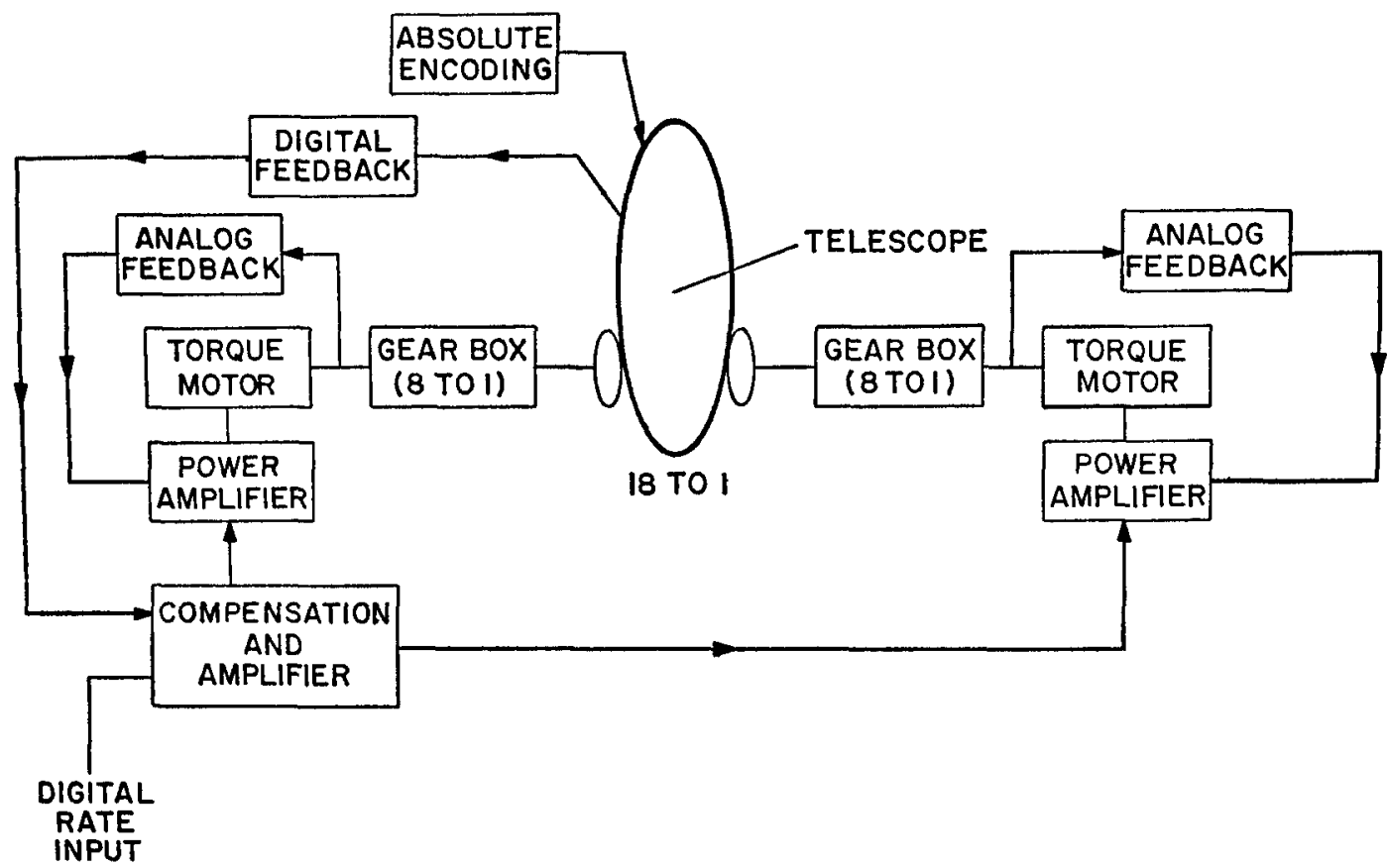

I5O inch TELESCOPE SERVO DRIVE BLOCK DIAGRAM

Fig. 1

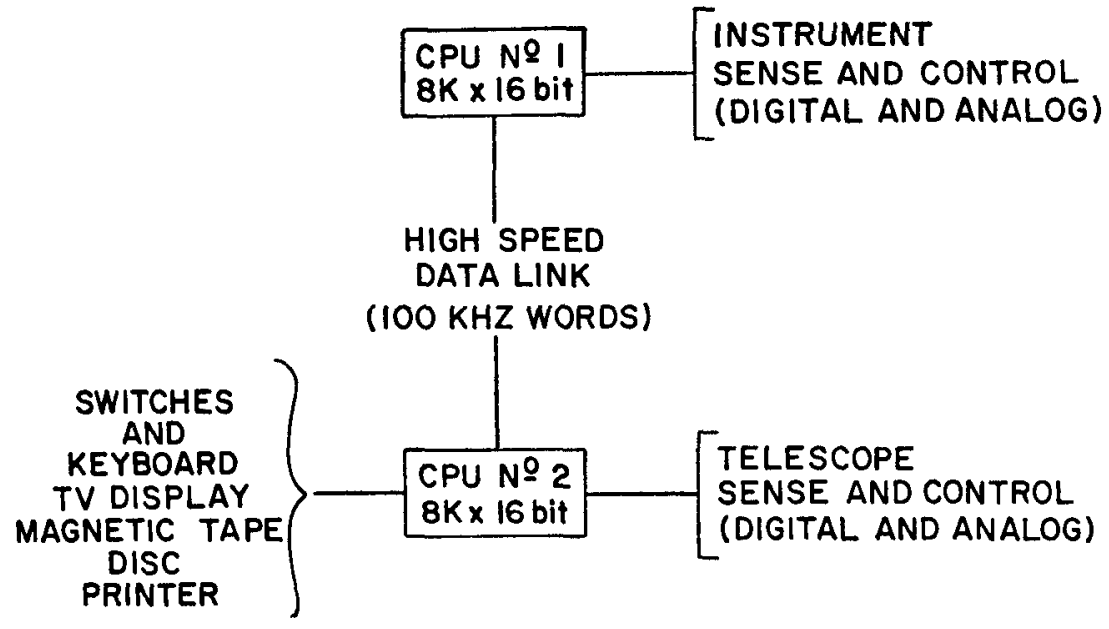

150 inch COMPUTER SYSTEM BLOCK DIAGRAM

Fig. 2 
Tololo was built with the drive of one astronomer primarily, with a number of people from the Observatory actively helping. At Tololo right now it's being maintained and kept going almost entirely by that same person, though almost all of the astronomers are doing software development and are helping out.

E. W. Densison: Our systems as far as I can tell, have universally turned in gains of the order of a factor of two or more in observing efficiency with a given telescope, etc. Our failures have in general been such that we lost fewer telescope hours through electronic failures than through mechanical failures, and our telescopes are not bad mechanically either. You can look forward to very high reliability in the electronic equipment.

W. A. SHERWOOD: How do you deal with flexure; do you compute it out or does the observer correct it out?

D. L. Crawford: At the present we don't handle any of the telescopes with a computer system. We plan, in the system described for the 150-inch, to handle flexure to the point that we can predict it and hence compute it out.

E. W. Dennison: Again we do not point the telescope at the moment. Obviously one can compute it. However, I'm thinking about trying to generate an experience look-up table based on data gathered by the system itself. I don't know what the magnitude of these flexure errors will be, but they must be very small. On the 200 -inch we have already found that with properly pre-computed values, taking into account aberration, refraction, and so on, we can use the system that's there, a synchro system, and we're able to come within 10 arcsec of any object we want in the sky. So already we have a basic system that's good to this kind of precision.

C. L. Stephens: Your slide showed two identical central processors at Cerro Tololo; was that for increased reliability?

D. L. CRAWFORD: They have a second central processor unit at Cerro Tololo entirely for redundancy, as a backup to the one in use.

R. B. DuNN: We have "power fail safe" on our installation, it's a very high interrupt, and when the power goes down this is the last thing that's turned on and you save the registers. There's a downfall in this, and we haven't been able to use it, because when you work with a large number of interrupts it turns out you also have to save the condition of the interrupts, or it's almost a useless feature. Later process controllers like the Xerox Sigma 3 do have this feature, but we haven't found "power fail safe" to be a useful device at all. Another point is that power supplies on both small and large computers fail to filter at very high frequencies. They work fine for the low frequencies, but high-frequency lightning glitches come sailing through the whole computer and take out the integrated circuits. We have to smooth the power with a motor-generator unit to really get a good enough atmosphere for a computer at a mountain observatory.

F. E. RoACH: What is the maximum practical distance between telescope and observer when automatic controls are used? Can the astronomer stay home and do his work?

D. L. CRAWFORD: We had for some years at Kitt Peak a remotely controlled telescope that operated on Kitt Peak from Tucson, but it had problems in the sense that it was designed and specified to solve certain problems that were basically incompatible with doing astronomy as we'd like to do it. It was not the fault of the communication systems. So with enough money one could stay home-depends where you put the money, and how much of it you have.

E. W. Dennison: I'd give the same answer, but 500 feet is a practical working limit without a large investment in special equipment. 